\title{
Selectivity for Conspecific Vocalizations within the Primate Insular Cortex
}

\author{
Rebecca Watson \\ Voice Neurocognition Laboratory, Department of Psychology, University of Glasgow, Glasgow G12 8QB, United Kingdom \\ Review of Remedios et al. (http://www.jneurosci.org/cgi/content/full/29/4/1034)
}

Successful processing of species-specific vocalizations is important for effective social interaction-not only for humans but for all vocal animals. However, until recently, little was known of the neural mechanisms involved in processing vocal information. A functional imaging study by Belin et al. (2000) revealed a voice-selective region in humans, located in the anterior of the temporal lobe, along the upper banks of the superior temporal sulcus. This region not only processes acoustical cues that distinguish our vocalizations from other sounds, but it is sensitive to auditory features that vary between human voices. This may allow us to identify different individuals. Yet, although face-selective visual regions have been found in both humans and monkeys, few investigations have examined whether analogous voice regions are present in nonhuman animals. Electrophysiological studies in monkeys have described neuronal responses to conspecific vocalizations, but comparison of these responses to those evoked by other sounds has widely been neglected and thus the significance hard to judge. Various electrophysiological studies (Tian et al., 2001) have reported some degree of voice selectivity in the early auditory cortical areas-for example, in the rostral lateral belt. In contrast, imaging studies have suggested that selective processing of vocal

Received March 27, 2009; revised April 23, 2009; accepted April 28, 2009. I thank Prof. Pascal Belin for his comments on this manuscript.

Correspondence should be addressed to Rebecca Watson, Voice Neurocognition Laboratory, Department of Psychology, Room 507, 58 Hillhead Street, University of Glasgow, Glasgow G12 8QB, UK. E-mail: r.watson@psy.gla.ac.uk. D01:10.1523/JNEUROSCI.1462-09.2009

Copyright $\odot$ 2009 Society for Neuroscience $\quad$ 0270-6474/09/296769-02\$15.00/0 sounds occurs in "higher-order" auditory areas, although the sites of these regions have varied from study to study, ranging from a region within the anterior superior temporal plane (Petkov et al., 2008) to the monkey homolog of language areas in humans (Gil-da-Costa et al., 2006). Recently, Recanzone (2008) investigated the discrepancy between electrophysiological and imaging studies by recording the responses of single neurons in core and belt auditory cortical fields to vocalizations. He observed no significant difference in the overall firing rate of neurons between these different cortical areas, thus disputing the claim that the early rostral cortical areas are preferentially involved in the selective processing of vocalizations. However, with the exception of a few studies (Cohen et al., 2004), electrophysiological evidence of selectivity to species-species vocalizations outside the auditory cortical areas is lacking.

Several studies, mainly using imaging techniques (e.g., fMRI), have implicated the insula in the processing of sound information, and in humans, the handling of speech information (Wong et al., 2004). Furthermore, lesions to the insula impair speech production (Habib et al., 1995). Still, this manner of investigation can only give, at best, indirect evidence of neuronal properties and mechanisms. By providing direct evidence of neuronal activity, electrophysiological studies can offer valuable information regarding the way in which selectivity to conspecific vocalizations arises, and this was the aim of a study conducted by Remedios et al. (2009), recently published in The Journal of Neuroscience. The purpose of their inves- tigation was to investigate the auditory response of macaque insula neurons, examine selectivity toward conspecific macaque vocalizations, and quantify both this selectivity and the level of discrimination between individual macaque vocalizations.

In this study, the insular neuronal activity of two macaque monkeys was investigated. The authors used a combination of anatomical magnetic resonance images and landmarks such as activity transitions between gray and white matter to estimate recording site and depth within the insula. The authors first used pure tones to compare the response properties of the insula neurons to those recorded from the primary and secondary auditory cortices. Acoustic responses within the insula were mainly restricted to an "auditory region," located within the mid to caudal extent of this structure. Insula neurons appeared to be less frequency selective and had significantly longer latencies than neurons in the auditory cortex. Moreover, in contrast to auditory-cortex neurons, the response pattern of the neurons in the insula did not reflect the stimulus's temporal structure.

Next, to investigate the insula's role in processing natural sounds and conspecific communication signals, the authors measured the response to sets of stimuli from three categories: macaque vocalizations [MVocs (coos, grunts, barks, pant-threats, and screams)], noises and vocalizations of other animals [AVocs (e.g., birds, lions, horses)], and environmental sounds [ESnds (e.g., wind, water, doors)]. Population and lifetime-selectivity indices were calculated to measure how these sounds were encoded 
by the insula. Insula neurons not only showed a significantly greater response to natural sounds (MVocs, AVocs, and ESnds) compared with those in the auditory cortex but also responded differentially to the three sound categories: there was a stronger response to MVocs than to either AVocs or ESnds, highlighting a preferential response for conspecific vocalizations. This was in direct contrast to the response of auditory neurons, whose response strength was significantly weaker for MVocs compared with AVocs and ESnds.

To ensure that results were not influenced by acoustical differences between sound categories, the authors probed insula neurons with a set of manipulated MVocs: specifically, one vocalization preserving the spectrum of the original vocalization but not its temporal envelope; one preserving the temporal envelope but not the spectrum; and one preserving both features but not their interaction. Results showed that insula neurons were indeed selective to the original conspecific vocalizations and preferred these over manipulations that contained only some of the acoustical features.

The authors then considered whether the macaques could differentiate between individual vocalizations, by calculating the neurons' response selectivity index (defined by the number of stimuli eliciting at least half a neuron's maximal response). They found that on average $33 \%$ of vocalizations elicited more than half the maximal response, indicating a greater selectivity of insula neurons compared with those in the superior temporal regions and auditory cortices, which together showed a range of $\sim 30-60 \%$. Finally, the authors asked whether insula neurons showed selectivity to call categories (in other words, call types carrying different behavioral meaning-for example, "coo" and "pant-threat"). Results indicated that this was not the case-many neurons responded strongly to one but not to other calls from one particular category, but at the same time also responded to one or two calls from a different category. This suggests that although insula neurons respond selectively to certain vocalizations, they do not necessarily represent categories of these vocalizations.

Auditory processing in macaque monkeys has been reasonably investigated, but there has been little exploration into the neural correlates of vocal processing in these animals: generally, studies of auditory processing in nonhuman primates have involved basic auditory stimuli such as pure tones or clicks. Only a few studies have used communication sounds in an attempt to in- vestigate voice-selectivity in nonhuman primates. Recently, researchers from the same group identified voice-selective regions in the macaque monkey brain, using fMRI as opposed to electrophysiological techniques (Petkov et al., 2008). Overall, the most reliable specificity for conspecific vocalizations was observed in the anterior auditory superior temporal plane region. This area lies in the proposed auditory "what" processing pathway, which is suggested to process the identity of sounds. In awake (as opposed to anesthetized) macaques, although, a strong preference for species-species vocalizations was also observed within the first few auditory cortical processing stages, including the primary field A1. This result, interestingly, contrasts with that of Remedios et al. (2009), where the response strength for MVocs in the auditory cortex was significantly weaker than the response for AVocs and ESnds. However, Petkov et al. (2008) acknowledge in their study that this region did show a less reliable preference for conspecific vocalizations, in addition to instability in location. The current electrophysiological data appears to clarify that we cannot attribute a consistent voice-selective response to this area.

The results of Remedios et al. (2009) offer a unique insight into the possible roles of the insula in the handling of vocal information. Despite support for the insula's contribution toward auditory processing, electrophysiological evidence of its contribution toward handling of vocal information in nonhuman primates is nonexistent. This study provides evidence that the insular auditory region processes more than just linguistic information, because the vocalizations of nonhuman primates-who lack the linguistic capabilities of humans - still evoke a significant response in this area. This prompts the question as to whether the insula may perform a comparable role in human subjects - specifically, the handling of particular vocal paralinguistic information. A few human imaging studies (Morris et al., 1999), using nonverbal vocalizations such as laughs and cries, have pointed to a possible role of insula in the processing of vocal emotion. Indeed, in the macaque, multiple interconnections to limbic structures such as the amygdala (Mesulam and Mufson, 1982) suggest the insula may well play a part in integrating vocal communication sounds and emotions. Studies on the regions involved in processing of (human) vocal identity are limited; however, there is converging evidence for a role of the anterior temporal lobe regions (part of the ventral what processing pathway). Again, with projections to temporal areas, it is plausible that the insula could also be involved in the handling of this information. The results of the study by Remedios et al. (2009) hold up such a claim, with insula neurons responding highly selectively within the set of vocalizations. This suggests that one role of these neurons could be to identify individual vocalizations. Whether such a response in macaques is also present in humans remains to be seen.

Overall, the results of Remedios et al. (2009) offer valuable insight into the neural correlates of vocalization processing in nonhuman primates, by providing electrophysiological evidence of the insula's involvement in the handling of conspecific communication sounds. The ongoing challenge will be not only to determine the exact role the insula plays in processing of vocal sound information but also to elucidate how such results may translate to human subjects. With this knowledge, we may be able to build on existing models of voice perception.

\section{References}

Belin P, Zatorre RJ, Lafaille P, Ahad P, Pike B (2000) Voice-selective areas in human auditory cortex. Nature 403:309-312.

Cohen YE, Russ BE, Gifford GW 3rd, Kiringoda R, MacLean KA (2004) Selectivity for the spatial and nonspatial attributes of auditory stimuli in the ventrolateral prefrontal cortex. J Neurosci 24:11307-11316.

Gil-da-Costa R, Martin A, Lopes MA, Muñoz M, FritzJB, Braun AR (2006) Species-specific calls activate homologs of Broca's and Wernicke's areas in the macaque. Nat Neurosci 9:1064-1070.

Habib M, Daquin G, Milandre L, Royere ML, Rey M, Lanteri A, Salamon G, Khalil R (1995) Mutism and auditory agnosia due to bilateral insular damage-role of the insula in human communication. Neuropsychologia 33:327-339.

Mesulam MM, Mufson EJ (1982) Insula of the old world monkey. III: Efferent cortical output and comments on function. J Comp Neurol 212:38-52.

Morris JS, Scott SK, Dolan RJ (1999) Saying it with feeling: neural responses to emotional vocalizations. Neuropsychologia 37:1155-1163.

Petkov CI, Kayser C, Steudel T, Whittingstall K, Augath M, Logothetis NK (2008) A voice region in the monkey brain. Nat Neurosci 11:367-374.

Recanzone GH (2008) Representation of conspecific vocalizations in the core and belt areas of the auditory cortex in the alert macaque monkey. J Neurosci 28:13184-13193.

Tian B, Reser D, Durham A, Kustov A, Rauschecker JP (2001) Functional specialization in rhesus monkey auditory cortex. Science 292:290-293.

Wong PC, Parsons LM, Martinez M, Diehl RL (2004) The role of the insular cortex in pitch pattern perception: the effect of linguistic contexts. J Neurosci 24:9153-9160. 